\title{
Identification of clinically relevant subgroups of COPD based on airway and circulating autoantibody profiles
}

\author{
ZHENYU LIANG $^{1 *}$, FEI LONG ${ }^{2 *}$, FENGYAN WANG $^{1 *}$, YUQIONG YANG $^{1}$, JING XIAO $^{2}$, \\ KUIMIAO DENG ${ }^{1}$, WEILI GU ${ }^{1}$, LUQIAN ZHOU ${ }^{1}$, JIAXING XIE ${ }^{1}$, WENHUA JIAN ${ }^{1}$, XIN CHEN ${ }^{3}$, \\ MEI JIANG ${ }^{1}$, JINPING ZHENG ${ }^{1}$, TAO PENG $^{2}$ and RONGCHANG CHEN ${ }^{1,4}$ \\ ${ }^{1}$ State Key Laboratory of Respiratory Disease, National Clinical Research Center for Respiratory Disease, \\ Guangzhou Institute of Respiratory Health, The First Affiliated Hospital of Guangzhou Medical University, \\ Guangzhou, Guangdong 510120; ${ }^{2}$ State Key Laboratory of Respiratory Disease, Sino-French Hoffmann Institute, \\ College of Basic Medical Science, Guangzhou Medical University, Guangzhou, Guangdong 511436; \\ ${ }^{3}$ Department of Respiratory Medicine, Zhujiang Hospital, Southern Medical University, Guangzhou, Guangdong 510280; \\ ${ }^{4}$ Shenzhen Institute of Respiratory Disease, Shenzhen People's Hospital, Shenzhen, Guangdong 518020, P.R. China
}

Received October 30, 2018; Accepted April 30, 2019

DOI: $10.3892 / \mathrm{mmr} .2019 .10498$

\begin{abstract}
Autoimmunity may serve a role in the pathological features of a subgroup of patients with chronic obstructive pulmonary disease (COPD); however, in immunological subgroups of COPD patients, the interrelationships between airway and circulating autoantibody responses, and clinical parameters, remain unclear. The present study was undertaken to evaluate these interrelationships in various immunological subgroups of COPD patients. Sputum supernatant and serum obtained from 102 patients with stable COPD were assayed for the presence of immunoglobulin $\mathrm{G}$ antibodies against ten autoantigens via Luminex multiplex technology. Hierarchical clustering based on principal components was performed on autoantibody profiles to classify patients into clusters. Network-based and module analyses were conducted
\end{abstract}

Correspondence to: Professor Rongchang Chen, State Key Laboratory of Respiratory Disease, National Clinical Research Center for Respiratory Disease, Guangzhou Institute of Respiratory Health, The First Affiliated Hospital of Guangzhou Medical University, 151 Yanjiang Road, Guangzhou, Guangdong 510120, P.R. China

E-mail: chenrc@vip.163.com

Professor Tao Peng, State Key Laboratory of Respiratory Disease, Sino-French Hoffmann Institute, College of Basic Medical Science, Guangzhou Medical University, Panyu Campus of Guangzhou Medical University, Building B, Guangyi Road, Guangzhou, Guangdong 511436, P.R. China

E-mail: pengtao@gzhmu.edu.cn

${ }^{*}$ Contributed equally

Key words: chronic obstructive pulmonary disease, autoantibody, unsupervised classification, network analysis, differential network analysis to explore interrelationships among autoantibodies and clinical variables in each cluster. Topological characteristics were compared between clusters. Unsupervised clustering identified four clusters: No significant differences in the majority of clinical characteristics were observed among clusters. In cluster 1, retrospective exacerbation was only positively associated with COPD assessment test score. Lung functions (predicted \% of forced expiratory volume in $1 \mathrm{sec}$ and maximal mid-expiratory flow) were negatively associated with exacerbation risk only in cluster 2. Sputum autoantibodies (against U1 small nuclear ribonucleoprotein, proteinase- 3 and Ro/Sjögren syndrome type A antigen) were negatively associated with exacerbation risks in cluster 2 , but positively associated in cluster 3 . The four networks also exhibited distinct topological properties. In COPD, autoantibody responses were heterogeneous and differentially associated with exacerbation risk in certain subgroups; their dual character should be considered in future research.

\section{Introduction}

Chronic obstructive pulmonary disease (COPD) is an important and growing cause of morbidity and mortality, and is predicted to be the third leading cause of mortality globally by 2020 (1). Patients with COPD can suffer from episodes of symptom exacerbations during the course of the disease that negatively affect their prognosis; however, COPD and its exacerbations are both heterogeneous conditions that are linked to complex and heterogeneous immune responses $(2,3)$.

Autoimmunity has been suggested to be an influential factor in the progression of patients who have suffered from COPD for $>10$ years $(4,5)$, as COPD shares numerous pathophysiological and clinical characteristics with autoimmune diseases (4). Increasing evidence indicates that autoimmune responses serve a role in the development and progression of COPD (6-10). Autoantibodies in stable COPD have been comprehensively reviewed recently (11); however, the 
heterogeneity of autoimmunity should also be considered. Kim et al (12) reported abnormal blood T-lymphocyte subsets in a subgroup of patients with COPD. Our recent study demonstrated that sputum autoantibody levels were associated with exacerbation risk in a subgroup of COPD patients (13), suggesting that autoimmunity is highly heterogeneous in COPD.

Network-based analysis is a novel integrative research approach that is suitable for the study of complex and heterogeneous conditions, such as COPD and its exacerbations (14-17). Divo et al (18) used network analysis to investigate the association between multiple comorbidities in patients with stable COPD. The authors included 79 comorbidities and various demographic, clinical and functional parameters in the network analysis, and observed that the comorbidities were significantly interlinked and formed a complex network in which six sub-networks (also termed modules) were identified. Grosdidier et al (19) used an integrative network-based approach to investigate the biological associations between COPD, its comorbidities and the chemical products contained in tobacco smoke. They revealed that comorbidities shared genes, proteins and biological pathways with COPD. Faner et al (20) explored the association between comorbidities and patients with exacerbated COPD from a molecular viewpoint (also termed a molecular diseasome) using network analysis. Noell et al (21) explored the pathobiological mechanisms of exacerbations and biomarkers by comparing multi-level (clinical, physiological, biological, imaging and microbiological) correlation networks determined during exacerbation and convalescence in patients with COPD; however, no known study has investigated the interrelationships between airway and circulating autoantibody responses, and clinical parameters in immunological subgroups of patients with COPD. It was hypothesised that network analysis, an analytical approach that involves the comparison of clinical, functional, biological and immunological correlation networks, may provide a novel insight into the complex association between autoantibody profiles and COPD clinical parameters. To properly adjust for the redundancy of autoantibody profiles and the heterogeneity of autoantibody responses, principal component analysis (PCA) and hierarchical clustering were performed prior to network analysis. Thus, in the present proof-of-concept study, network analysis based on unsupervised classification was used to: i) Compare the network structures of different COPD subgroups identified by sputum and serum autoantibody profiles; and ii) identify a series of exacerbation risk-associated factors.

\section{Materials and methods}

Patients. This was a prospective cross-sectional study. A total of 102 patients with COPD with stable disease were enrolled at the First Affiliated Hospital of Guangzhou Medical University (Guangzhou, China) between March 2017 and October 2017. A group of 18 non-smoking healthy controls was also enrolled for comparison. Inclusion criteria for patients with COPD were: i) Aged $>40$ years; and ii) confirmed diagnosis of COPD according to the Global Initiative for Chronic Obstructive Lung Disease (GOLD) guidelines (22) [post-bronchodilator forced expiratory volume in $1 \mathrm{sec}$ (FEV1)/forced vital capacity (FVC) ratio <0.7]. Exclusion criteria were: i) Diagnosis of known respiratory disorders other than COPD; ii) history of significant inflammatory disease other than COPD; iii) COPD exacerbation within 4 weeks of enrolment; iv) history of lung surgery and tuberculosis; v) diagnosis of cancer; vi) having undergone a blood transfusion within 4 weeks of enrolment; vii) diagnosis of autoimmune diseases; and viii) enrolment in a blinded drug trial. The clinicopathological data of the patients and healthy controls are presented in Table I.

Inclusion criteria for non-smoking healthy controls were: i) Aged >40 years; and ii) without any known respiratory disorders and significant inflammatory diseases. Subjects with one or more of the following criteria were excluded: i) Diagnosis of known respiratory diseases; ii) history of significant inflammatory disease; iii) diagnosis of cancer; iv) blood transfusion within 4 weeks of enrolment; v) inability to walk; or vi) current participation in an intervention trial.

Written informed consent was obtained from all patients. The study was approved by the ethics committee of the First Affiliated Hospital of Guangzhou Medical University (permit no. 2017-22) and was registered with www.clinicaltrials.gov (NCT 03240315).

Clinical and functional parameters. Data collected at enrolment included demographic characteristics, lung function, COPD assessment test (CAT), and modified Medical Research Council Dyspnea Scale (mMRC) of subjects prior to sputum induction. Spirometry was performed according to the American Thoracic Society guidelines (23).

Blood samples, sputum collection and processing. Peripheral venous blood samples ( $4 \mathrm{ml}$ per subject) were collected into a vacuum tube, and serum was obtained by centrifuging whole blood at 1,057 x g (3,000 rpm) for $10 \mathrm{~min}$ at room temperature. Sputum induction was performed according to guidelines suggested by the Task Force of the European Respiratory Society (24). A two-step procedure was conducted to process the sputum as previously described (25). Sputum supernatant and serum were stored at $-80^{\circ} \mathrm{C}$.

Autoantibody detection. Based on a literature search, ten autoantigens with known or putative links to COPD were selected (26-29), including Smith antigen (Sm), ribosomal phosphoprotein $\mathrm{P0}$ (P0), Ro/Sjögren syndrome type A antigen (SS-A), La/Sjögren syndrome type B antigen (SS-B), DNA topoisomerase I (Scl70), histidyl-tRNA synthetase (Jo1), U1 small nuclear ribonucleoprotein (U1-SnRNP), thyroid peroxidase (TPO), proteinase-3 (PR3) and myeloperoxidase (MPO). Autoantigens (DIARECT AG) were coupled with multiplex magnetic beads (Bio-Rad Laboratories, Inc.) and incubated with sputum supernatant and serum samples diluted 1:10 and 1:180, respectively, at $37^{\circ} \mathrm{C}$ for $1 \mathrm{~h}$. The beads were washed using the Bio-Plex Pro ${ }^{\mathrm{TM}}$ wash station (Bio-Rad Laboratories, Inc.), and then incubated at $37^{\circ} \mathrm{C}$ for $1 \mathrm{~h}$ with biotin-conjugated anti-human IgG (1:1,000; cat. no. A24474; Thermo Fisher Scientific, Inc.). Subsequently, they were washed and then reacted for $15 \mathrm{~min}$ at $37^{\circ} \mathrm{C}$ with streptavidin-R-phycoerythrin (Bio-Rad Laboratories, Inc.). After the microspheres were washed and resuspended, the median fluorescence intensity of each encoded microsphere was measured using Bio-Plex 200 with an excitation wavelength at $532 \mathrm{~nm}$ and emission 
Table I. Subject demographics and clinical characteristics.

\begin{tabular}{|c|c|c|}
\hline Characteristic & $\begin{array}{l}\text { Non-smoking healthy } \\
\text { controls, } n=18\end{array}$ & $\begin{array}{c}\text { COPD patients, } \\
\mathrm{n}=102\end{array}$ \\
\hline Age, years & $58.33 \pm 7.67$ & $66.46 \pm 8.10$ \\
\hline $\operatorname{Sex}(M / F)$ & $10 / 8$ & $98 / 4$ \\
\hline BMI, $\mathrm{kg} / \mathrm{m}^{2}$ & $25.26 \pm 3.65$ & $21.86 \pm 4.11$ \\
\hline $\begin{array}{l}\text { Smoking, } \mathrm{n} \\
\text { (never/ex/current) }\end{array}$ & $18 / 0 / 0$ & $10 / 73 / 19$ \\
\hline Pre-BD FEV1, litres & $2.51 \pm 0.79$ & $1.27 \pm 0.57$ \\
\hline Pre-BD FEV1pred\% & $96.94 \pm 16.79$ & $49.02 \pm 21.40$ \\
\hline Pre-BD FVC, litres & $3.15 \pm 0.98$ & $2.57 \pm 0.73$ \\
\hline Pre-BD FEV1/FVC & $0.80 \pm 0.06$ & $0.49 \pm 0.13$ \\
\hline Post-BD FEV1, litres & ND & $1.40 \pm 0.59$ \\
\hline Post-BD FEV1pred $\%$ & ND & $53.34 \pm 22.41$ \\
\hline Post-BD FVC, litres & ND & $2.74 \pm 0.73$ \\
\hline Post-BD FEV1/FVC & ND & $0.51 \pm 0.14$ \\
\hline CAT score & NA & $11.67 \pm 6.44$ \\
\hline mMRC & NA & $2(1-2)$ \\
\hline \multicolumn{3}{|l|}{$\begin{array}{l}\text { Respiratory } \\
\text { medications }\end{array}$} \\
\hline ICS & NA & $66(64.7 \%)$ \\
\hline LABA & NA & $66(64.7 \%)$ \\
\hline LAMA & NA & $41(40.2 \%)$ \\
\hline \multicolumn{3}{|c|}{$\begin{array}{l}\text { Data are presented as } \mathrm{n}(\%) \text {, mean } \pm \text { standard deviation or median } \\
\text { (interquartile range) unless otherwise stated. COPD, chronic obstruc- } \\
\text { tive pulmonary disease; pre-BD, pre-bronchodilator; post-BD, } \\
\text { post-bronchodilator; BMI, body mass index; FEV1, forced expira- } \\
\text { tory volume in } 1 \mathrm{sec} \text {; FVC, forced vital capacity; FEV1pred } \% \text {, forced } \\
\text { expiratory volume in } 1 \mathrm{sec} \text { as percentage of predicted; FVCpred\%, } \\
\text { forced vital capacity as percentage of predicted; CAT, COPD assess- } \\
\text { ment test; mMRC, Modified Medical Research Council Dyspnea } \\
\text { Scale; ICS, inhaled corticosteroids; LABA, long-acting } \beta \text { agonist; } \\
\text { LAMA, long-acting muscarinic antagonist; ND, no data; NA, not } \\
\text { applicable. }\end{array}$} \\
\hline
\end{tabular}

wavelength at $575 \mathrm{~nm}$ (Bio-Rad Laboratories, Inc.). Bio-Plex Manager $^{\mathrm{TM}} 6.0$ software (Bio-Rad Laboratories, Inc.) was used to generate the result files.

Statistical analysis. All statistical analyses were performed using SPSS software (version 19.0; IBM Corp.). PCA was performed on autoantibody profiles in sputum and serum, and components with eigenvalues $>1$ were extracted. Unsupervised agglomerative hierarchical clustering was performed on the above components, using the un-centred correlation as the similarity metric (Cluster version 3.0) (30). The dendrogram and resulting heatmap were visualised using TreeView (version 1.60) (31). Shapiro-Wilk test was performed to access the normality of distribution of each continuous variable, and depending on the distribution of the data, ANOVA or Kruskal-Wallis test were used to compare the clusters. Then, Fisher's Least Significant Difference (LSD) test or the Nemenyi test was performed to analyse the differences between clusters. Correlation networks integrating 45 clinical and molecular parameters were then established using Gephi software (version 0.9.1) (32). Networks integrating clinical and autoantibody parameters in each group were constructed using Spearman's correlation test. Correlation coefficients with $\mathrm{P}>0.05$ were excluded. Network clustering was conducted using the 'fast unfolding' algorithm within the Gephi software.

\section{Results}

Patient information. The clinical characteristics of 102 patients with COPD and 18 non-smoking healthy controls are presented in Table I. The mean ages of the patients and controls were $66.46 \pm 8.10$ and $58.33 \pm 7.67$ years, respectively.

Hierarchical clustering based on PCA. Sputum and serum autoantibody profile data were processed with PCA: The eight largest principal components extracted were able to account for $69.54 \%$ of the variability contained in the original data (Fig. S1; Table SI), suggesting that these eight components alone contributed to the majority of the information among the groups. Components and coefficient sets used in the analysis are presented in Table SII. Using hierarchical cluster analysis, four clusters of patients with COPD were identified based on the above components (Fig. 1).

Clinical characteristics of the four clusters. To determine whether the patients within these clusters represented clinically distinct subgroups of COPD, the clinical parameters of the four clusters were analysed (Table II). The average CAT score and mMRC of individuals in Cluster 2 were significantly increased compared with those in Cluster 4. Conversely, there were no significant differences in various other clinical characteristics [age, number of exacerbations in the previous year (AE), FEV1, FEV1 as a percentage of the predicted value (FEV1pred\%), maximal mid-expiratory flow (MMEF), and body mass index (BMI)] among clusters. Autoantibody levels of the four subtypes were also analysed (Figs. S2 and S3; Table SIII).

Differential network analysis. Fig. 2 presents the Spearman correlation networks integrating clinical and autoantibody parameters in healthy controls (Fig. 2A) and the aforementioned four clusters (Fig. 2B-E). Table III presents the comparisons of the topological properties of the five groups. Notable observations included: i) The five networks exhibited different topological properties (the cluster 2 network displayed high density, whereas the cluster 1 network displayed low density); ii) there were seven modules in cluster 1 , five modules in clusters 2 and 3 , and six modules in cluster 4, but all modules appeared markedly heterogeneous in their clinical and biological content, as the majority contained nodes of distinct functional and immunological categories (Fig. 2); and iii) the retrospective exacerbation-associated factors (AE-nodes in Fig. 2) were significantly different among the four clusters. In cluster 1 (Fig. 2B), the AE was only positively associated with the CAT score. In cluster 2 (Fig. 2C), the AE was negatively associated with age, lung function (FEV1pred\% and MMEF), sputum autoantibodies (P0, Scl70, Sm, U1-SnRNP, PR3 and Ro/SSA) and serum globulin (Glb), and positively associated with blood cell counts (peripheral 


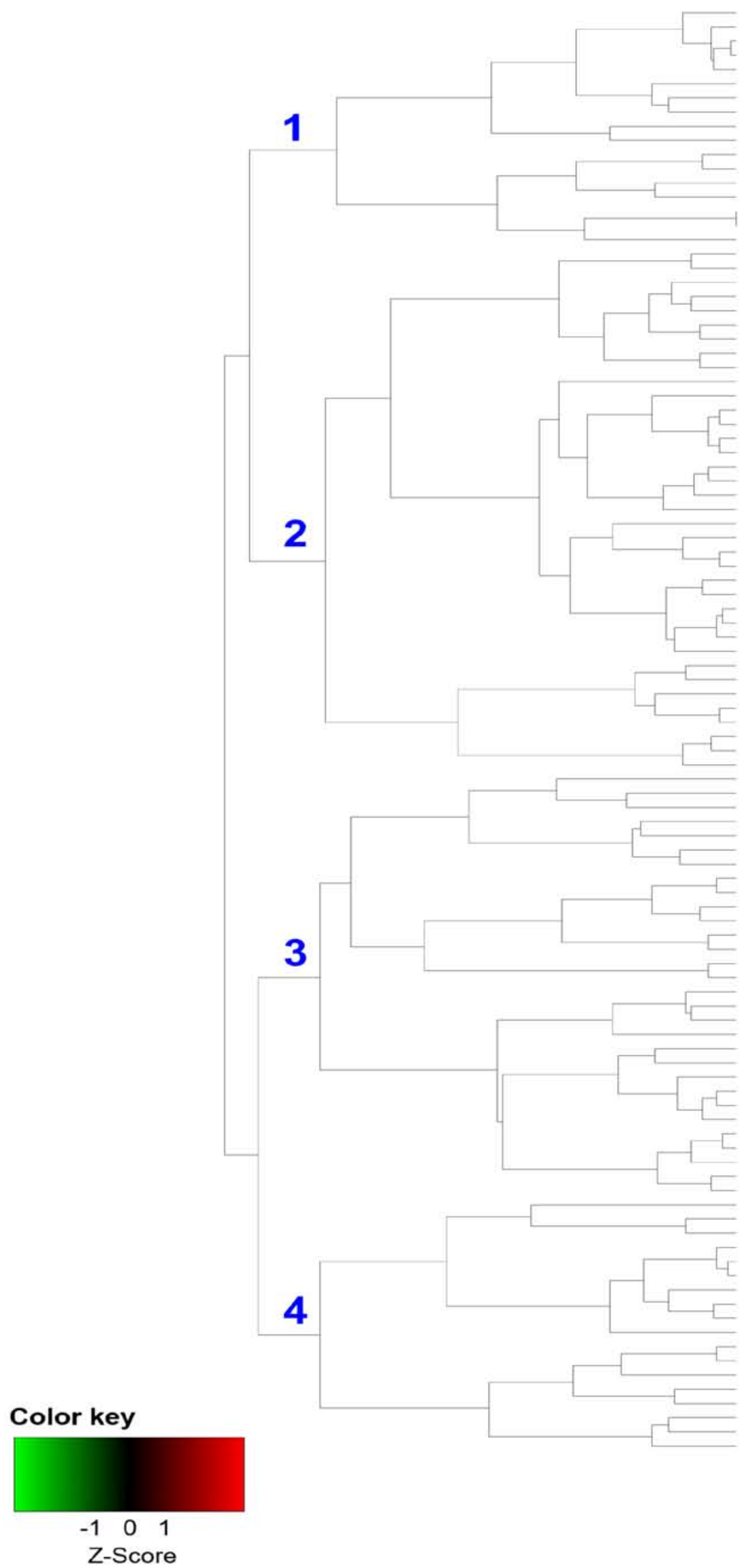$$
\text { Б' }
$$

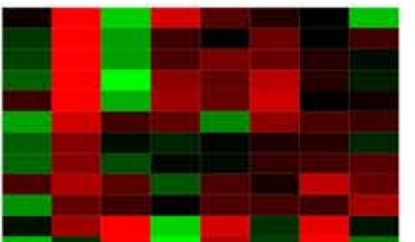

17
3
36

47

55

30
78

44
83

83
14

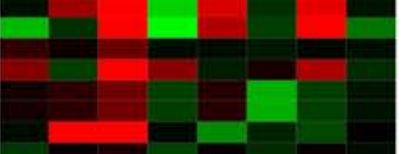

14
82

82
20
48

89
8

8
86
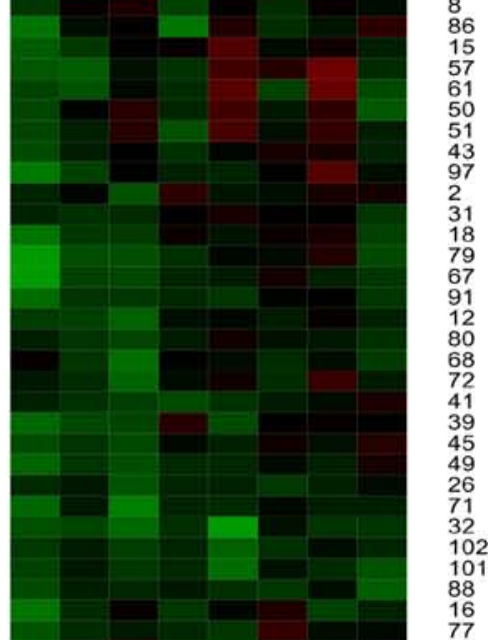

102

88

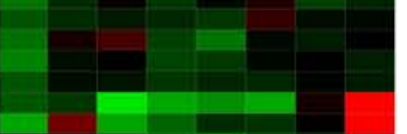

77
23

23
63

63
84
53
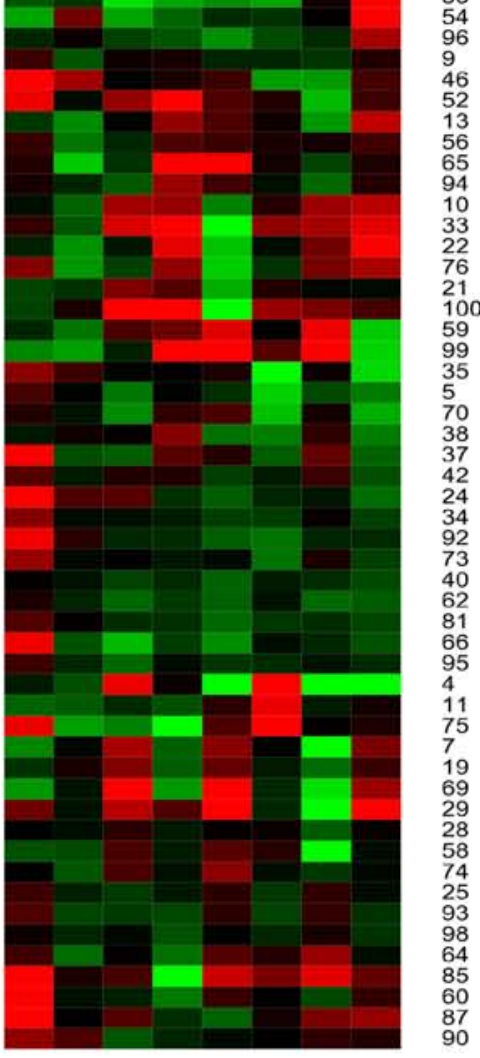

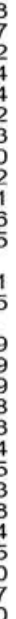

Figure 1. Hierarchical clustering based on principal component analysis. Each column is a component; each row is an individual patient. Numbers at the right side of the heat map indicate the patient number. Left, dendrogram presenting the similarity of groups; four clusters are indicated. 
Table II. Comparison of clinical parameters among clusters.

\begin{tabular}{lccccr}
\hline Clinical parameter & Cluster 1, $\mathrm{n}=17$ & Cluster 2, $\mathrm{n}=37$ & Cluster 3, $\mathrm{n}=30$ & Cluster 4, $\mathrm{n}=18$ & P-value \\
\hline Age, years & $66.65 \pm 8.21$ & $66.95 \pm 8.58$ & $67.03 \pm 8.40$ & $64.33 \pm 6.67$ & 0.681 \\
BMI, $\mathrm{kg} / \mathrm{m}^{2}$ & $21.76 \pm 5.62$ & $22.47 \pm 3.63$ & $20.79 \pm 4.07$ & $22.49 \pm 3.37$ & 0.355 \\
Number of exacerbations in & $0(0-1)$ & $1(0-1.5)$ & $1(0-2)$ & $0(0-1)$ & 0.109 \\
the previous year & & & & \\
Blood neutrophil count, $\times 109 / 1$ & $4.4 \pm 1.11$ & $4.76 \pm 2.45$ & $4.04 \pm 1.71$ & $4.46 \pm 1.69$ & 0.527 \\
FEV1pred\% & $45.16 \pm 18.72$ & $43.65 \pm 17.25$ & $53.26 \pm 24.11$ & $56.63 \pm 24.46$ & 0.095 \\
MMEF & $0.43 \pm 0.25$ & $0.46 \pm 0.30$ & $0.62 \pm 0.49$ & $0.68 \pm 0.46$ & 0.105 \\
CAT & $9.88 \pm 4.85$ & $13.84 \pm 6.90$ & $11.80 \pm 5.46$ & $8.67 \pm 7.01$ & $0.022^{\mathrm{a}}$ \\
mMRC & $1(1-2)$ & $2(1-2)$ & $1(1-2.25)$ & $1(0-2)$ & $0.029^{\mathrm{a}}$ \\
\hline
\end{tabular}

${ }^{\mathrm{a}} \mathrm{P}<0.05$; cluster 2 vs. cluster 4. BMI, body mass index; CAT, chronic obstructive pulmonary disease assessment test score; FEV1pred\%, forced expiratory volume in $1 \mathrm{sec}$ as percentage of predicted; MMEF, maximal mid-expiratory flow; mMRC, modified Medical Research Council Dyspnea Scale.

white blood cell, neutrophil and monocyte) and blood haemoglobin. In cluster 3 (Fig. 2D), the AE was positively associated with the CAT score and sputum autoantibodies (U1-SnRNP, PR3, MPO and Ro/SSA), and in cluster 4 (Fig. 2E), the AE was negatively associated with serum uric acid and blood neutrophil count (Table III). Additionally, sputum anti-PR3, sputum anti-Ro/SSA, and sputum anti-U1-SnRNP were significantly negatively correlated with AE in cluster 2 (Fig. 2C), but were positively correlated with AE in cluster 3 (Fig. 2D; Table III). The network of non-smoking controls had a lower density, lower average path length, lower average degree and longer diameter than those in COPD groups, reflecting normal immunological condition (Fig. 2A; Table III).

Exacerbation-related module analysis. In each cluster, network clustering yielded modules, and the module containing retrospective exacerbation (AE-node) was extracted for further analysis (Fig. 3). Table IV presents the topological properties of the four AE modules of the corresponding clusters. These modules exhibited significant heterogeneity in terms of their biological and clinical contents, in addition to their topological properties. Module 2 demonstrated very high network density, whereas module 4 demonstrated low density. Module 2 contained 13 nodes with a high average degree, whereas module 1 contained only three nodes with a low average degree. In module $1, \mathrm{AE}$ was only positively associated with the CAT score. In module 2, AE was negatively associated with sputum autoantibodies (P0, Scl70, Sm, U1-SnRNP, PR3 and Ro/SSA) and Glb, and positively associated with neutrophil counts. In module 3 , AE was positively associated with the CAT score and sputum autoantibodies (U1-SnRNP, PR3, MPO and Ro/SSA). In module 4, AE was negatively associated with serum uric acid (Fig. 3, Table IV). Sputum anti-PR3, sputum anti-Ro/SSA and sputum anti-U1-SnRNP were significantly negatively correlated with $\mathrm{AE}$ in module 2 , but were positively correlated with $\mathrm{AE}$ in module 3.

\section{Discussion}

Autoimmune components in COPD have received increasing attention, as COPD shares various pathophysiological and clinical characteristics with autoimmune diseases $(4,8,10,11)$; however, there remains a lack of medical literature regarding the relationship between airway/circulating autoantibody responses and clinical parameters in COPD, particularly in different heterogenous subgroups. In the present proof-of-concept study, three methods were employed to investigate the interrelationships among autoantibody profiles and clinical variables in various COPD subgroups. First, a highly sensitive detection method was used to simultaneously investigate autoantibody profiles in sputum and serum. Second, unsupervised clustering was performed on the PCA-transformed autoantibody profile data, independent of clinical parameters, to identify immunological subgroups of COPD. Third, a network-based analysis was applied to investigate the association between immunological and clinical parameters, and COPD exacerbation risks, in each cluster, followed by comparison of the networks and module properties of these clusters. The following main findings were reported: i) Four stable COPD subgroups with distinguished immunological features were identified, although there were no significant differences among subgroups for the majority of clinical characteristics; ii) the networks of the four subgroups exhibited distinct topological properties; iii) the exacerbation risk-associated factors were significantly different among the four clusters; and iv) sputum anti-PR3, sputum anti-Ro/SSA and sputum anti-U1-SnRNP were significantly negatively associated with exacerbation risk in cluster 2 , but positively associated in cluster 3 , suggesting the heterogeneity and dual nature of the airway autoantibody responses in COPD.

A number of previous studies have investigated autoantibodies in COPD from a clinical point of view. For example, Cheng et al (33) detected circulating IgG, IgA and IgM against human bronchial epithelial cells (anti-HBEC) in stable patients with COPD using indirect immunofluorescence, and observed an increased positive rate of anti-HBEC expression in patients with COPD compared with in healthy controls. Sigari et al (34) reported increased serum levels of anti-cyclic citrullinated peptide antibody levels in wood-smoke-induced COPD compared with in tobacco-induced COPD and controls. Xiong et al (35) reported that the plasma autoantibody levels of 
A
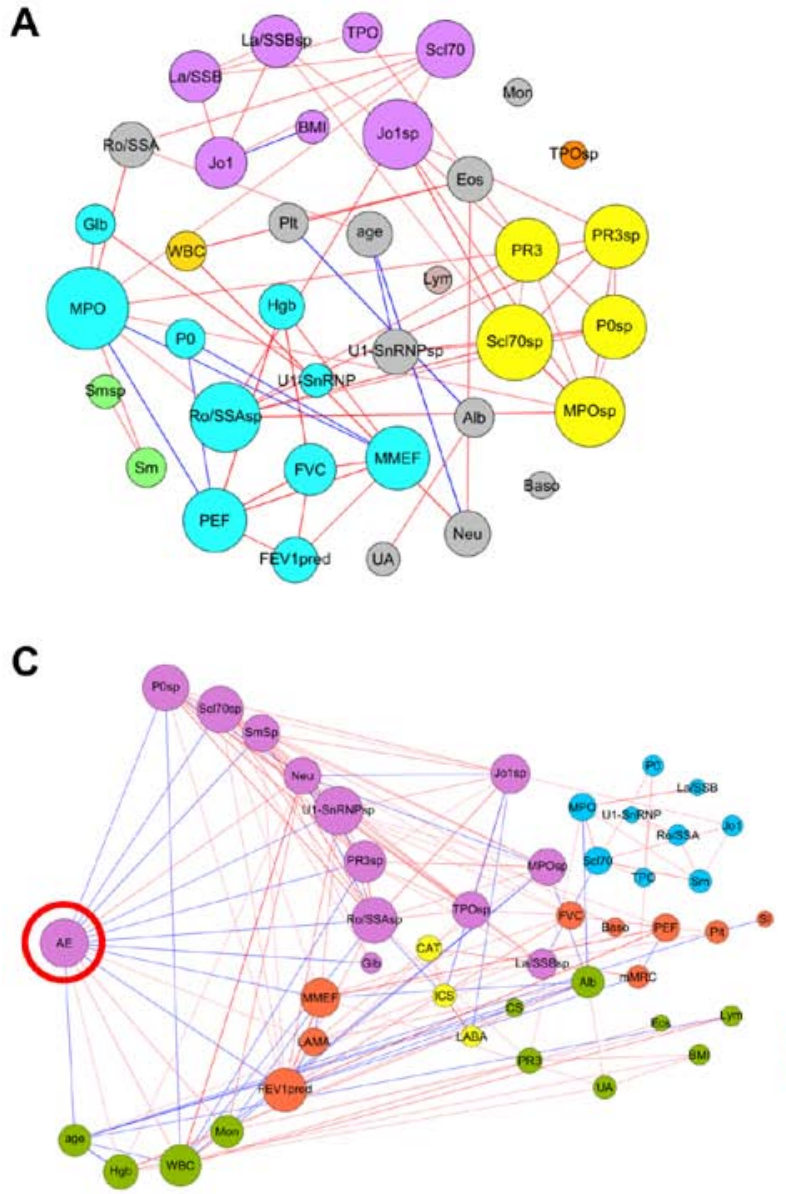

E

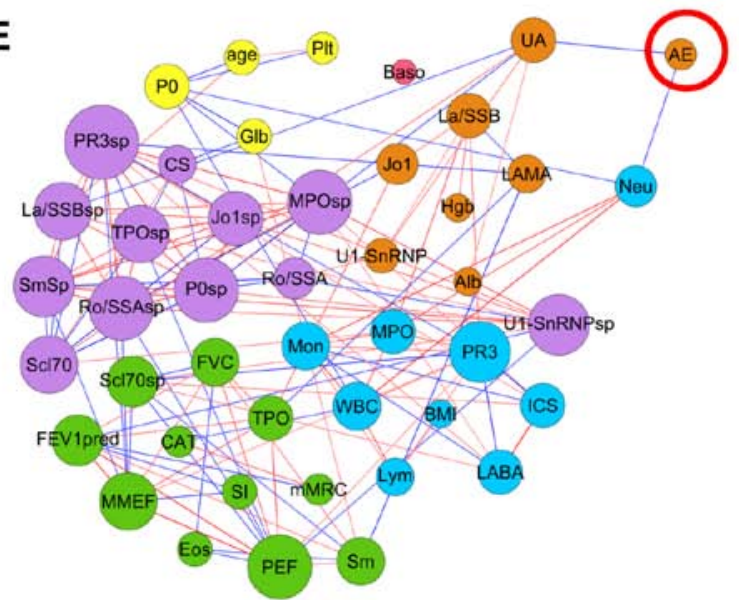

B

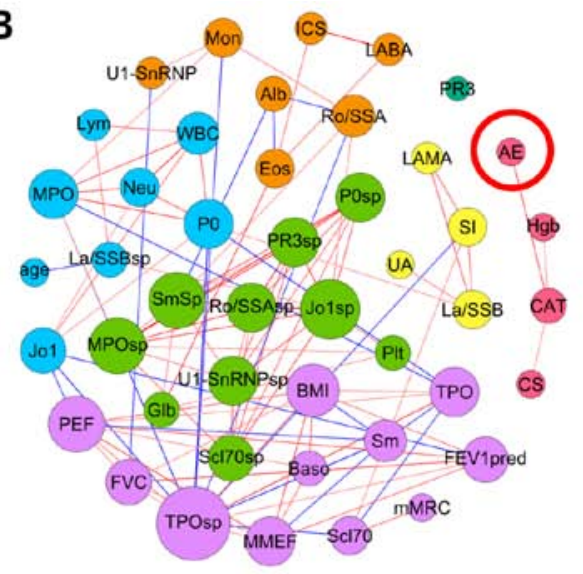

D

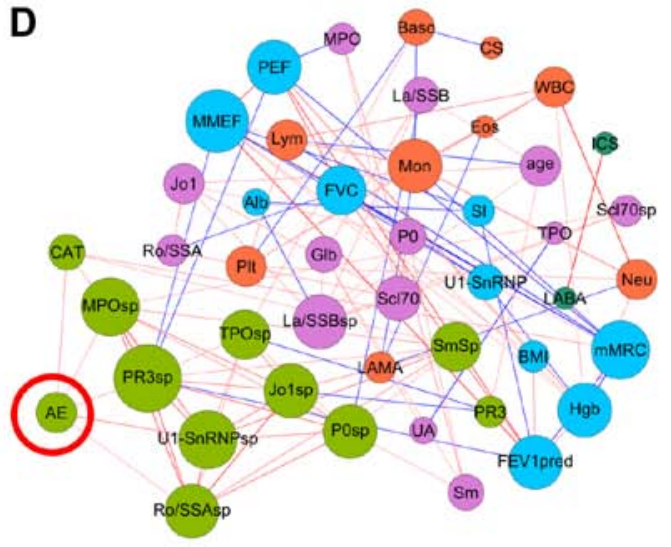

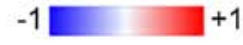

Edge weight $(r)$

Figure 2. Network analysis of non-smoking healthy controls and the four clusters. (A) Non-smoking healthy controls and clusters (B) 1, (C) 2 , (D) 3 and (E) 4 . The size of each node is proportional to its weighted degree value. The colour of each node represents the corresponding module. Correlation coefficients with $\mathrm{P}>0.05$ were filtered out. The colour of each edge indicates the correlation coefficient (edge weight) between two nodes. AE, number of exacerbations in the previous year; BMI, body mass index; Alb, serum albumin; CAT, chronic obstructive pulmonary disease assessment test score; FEV1pred, forced expiratory volume in $1 \mathrm{sec}$ as percentage of predicted; CS, current smoker; Hgb, haemoglobin; Glb, serum globulin; MMEF, maximal mid-expiratory flow; mMRC, modified Medical Research Council Dyspnea Scale; Mon, peripheral blood monocyte count; Neu, peripheral blood neutrophil count; sp, sputum; UA, serum uric acid; WBC, peripheral white blood cell count; Sm, Smith antigen; P0, ribosomal phosphoprotein P0; Ro/SSA, Ro/Sjögren syndrome type A antigen; $\mathrm{La} / \mathrm{SSB}, \mathrm{La} / \mathrm{Sjögren}$ syndrome type B antigen; Scl70, DNA Topoisomerase I; Jo1, histidyl-tRNA synthetase; U1-SnRNP, U1 small nuclear ribonucleoprotein; TPO, thyroid peroxidase; PR3, proteinase-3; MPO, myeloperoxidase.

IgG, IgA and IgM against cytokeratin-18 and -19 were elevated in patients with COPD compared with healthy controls. Luo et al (36) investigated the presence of anti-CD80 autoantibodies in the serum of patients with stable COPD and controls, and observed that serum levels of anti-CD80 were increased in patients with COPD compared with those in controls and were positively correlated with serum levels of interleukin (IL)-6 and IL-8. Shindi et al (37) detected serum IgM and IgG autoantibodies in patients with COPD and controls using an antigen microarray, and reported significant differences in the 
Table III. Topological properties of the four correlation networks.

A, Network properties

\begin{tabular}{|c|c|c|c|c|c|}
\hline Factor & $\begin{array}{l}\text { Non-smoking } \\
\text { healthy controls }\end{array}$ & Cluster 1 & Cluster 2 & Cluster 3 & Cluster 4 \\
\hline Number of nodes & 37 & 45 & 45 & 45 & 45 \\
\hline Average degree & 3.459 & 4.711 & 6.711 & 5.156 & 6.311 \\
\hline Number of edges & 64 & 106 & 151 & 116 & 142 \\
\hline Network diameter & 9 & 7 & 6 & 8 & 6 \\
\hline Graph density & 0.096 & 0.107 & 0.153 & 0.117 & 0.143 \\
\hline Average path length & 3.64 & 3.043 & 2.555 & 2.908 & 2.552 \\
\hline $\begin{array}{l}\text { Average Clustering } \\
\text { coefficient }\end{array}$ & 0.45 & 0.533 & 0.46 & 0.441 & 0.45 \\
\hline Modularity & 0.535 & 0.56 & 0.367 & 0.582 & 0.447 \\
\hline Module number & 10 & 7 & 5 & 5 & 6 \\
\hline $\begin{array}{l}\text { Hubs (nodes with } \\
\text { degree within top } 10 \% \text { ) }\end{array}$ & $\begin{array}{l}\text { Serum } \\
\text { anti-MPO, } \\
\text { sputum } \\
\text { anti-Scl70, } \\
\text { sputum } \\
\text { anti-MPO, } \\
\text { sputum } \\
\text { anti-Jo1, } \\
\text { sputum } \\
\text { anti-Ro/SSA }\end{array}$ & $\begin{array}{l}\text { Sputum } \\
\text { anti-TPO, } \\
\text { sputum } \\
\text { anti-Jo1, } \\
\text { sputum } \\
\text { anti-Sm, } \\
\text { sputum } \\
\text { anti-MPO, } \\
\text { PEF }\end{array}$ & $\begin{array}{l}\text { Sputum anti-U1- } \\
\text { SnRNP, } \\
\text { AE, sputum } \\
\text { anti-P0, } \\
\text { sputum } \\
\text { anti-Ro/SSA, } \\
\text { sputum } \\
\text { anti-Scl70 }\end{array}$ & $\begin{array}{l}\text { Sputum anti-PR3, } \\
\text { MMEF, mMRC, } \\
\text { sputum } \\
\text { anti-MPO, } \\
\text { sputum } \\
\text { anti-U1-SnRNP }\end{array}$ & $\begin{array}{l}\text { Sputum } \\
\text { anti-PR3, } \\
\text { sputum } \\
\text { anti-P0, } \\
\text { sputum } \\
\text { anti-Ro/ } \\
\text { SSA, } \\
\text { sputum } \\
\text { anti-MPO, } \\
\text { PEF }\end{array}$ \\
\hline
\end{tabular}

B, AE-node properties

\begin{tabular}{|c|c|c|c|c|c|}
\hline Factor & $\begin{array}{l}\text { Non-smoking } \\
\text { healthy controls }\end{array}$ & Cluster 1 & Cluster 2 & Cluster 3 & Cluster 4 \\
\hline Degree & NA & 1 & 15 & 5 & 2 \\
\hline Betweenness centrality & NA & 0 & 70.59 & 3.59 & 7.47 \\
\hline Eccentricity & NA & 2.0 & 5 & 5 & 5.0 \\
\hline Closeness centrality & NA & 0.6 & 0.49 & 0.35 & 0.32 \\
\hline Clustering coefficient & NA & 0 & 0.41 & 0.7 & 0 \\
\hline Correlated nodes & NA & $\begin{array}{l}\text { Positive: CAT } \\
\text { Negative: none }\end{array}$ & $\begin{array}{l}\text { Positive: Neu, Mon, } \\
\text { WBC, Hgb } \\
\text { Negative: FEV1 pred\%, } \\
\text { MMEF, sputum anti-P0, } \\
\text { sputum anti-Scl70, } \\
\text { sputum anti-Sm, sputum } \\
\text { anti-U1-SnRNP, sputum } \\
\text { anti-PR3, sputum } \\
\text { anti-Ro/SSA, Glb, age }\end{array}$ & $\begin{array}{l}\text { Positive: CAT, } \\
\text { sputum anti-PR3, } \\
\text { sputum anti-MPO, } \\
\text { sputum anti-Ro/SSA, } \\
\text { sputum anti-U1-SnRNP } \\
\text { Negative: none }\end{array}$ & $\begin{array}{l}\text { Positive: } \\
\text { none } \\
\text { Negative: } \\
\text { UA, Neu }\end{array}$ \\
\hline
\end{tabular}

AE, number of exacerbations in the previous year; FEV1pred\%, forced expiratory volume in $1 \mathrm{sec}$ as percentage of predicted; MMEF, maximal mid-expiratory flow; Neu, peripheral blood neutrophil count; CAT, chronic obstructive pulmonary disease assessment test score; WBC, peripheral white blood cell count; Glb, serum globulin; Mon, peripheral blood monocyte count; Hgb, haemoglobin; UA, serum uric acid; Sm, Smith antigen; P0, ribosomal phosphoprotein P0; Ro/SSA, Ro/Sjögren syndrome type A antigen; Scl70, DNA topoisomerase I; Jo1, histidyl-tRNA synthetase; U1-SnRNP, U1 small nuclear ribonucleoprotein; TPO, thyroid peroxidase; PR3, proteinase-3; MPO, myeloperoxidase.

autoantigenic specificities of $\operatorname{IgM}$ autoantibodies compared with IgG autoantibodies in COPD serum. Conversely, none of these studies reported airway autoantibody responses in
COPD, and no studies have investigated the autoantibody responses in different immunological subgroups of COPD. Therefore, the present study was conducted to investigate the 
A

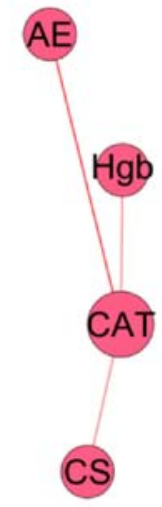

C

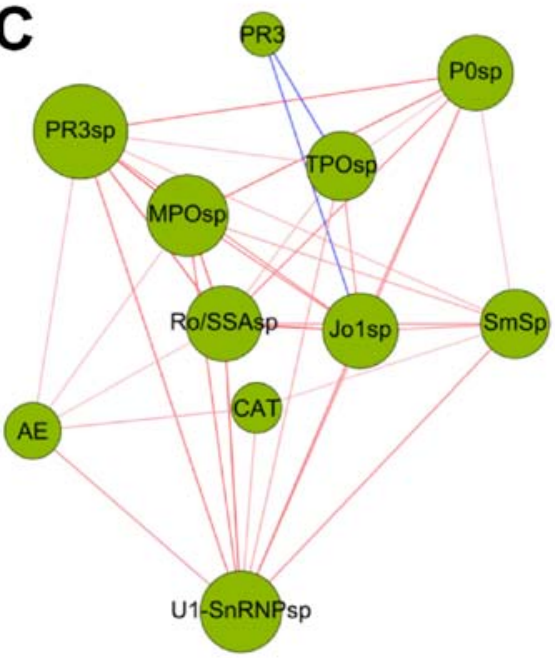

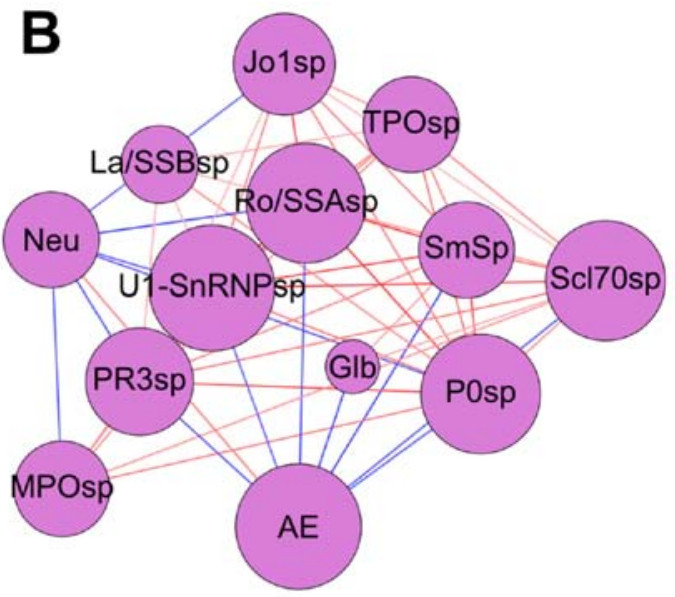

D

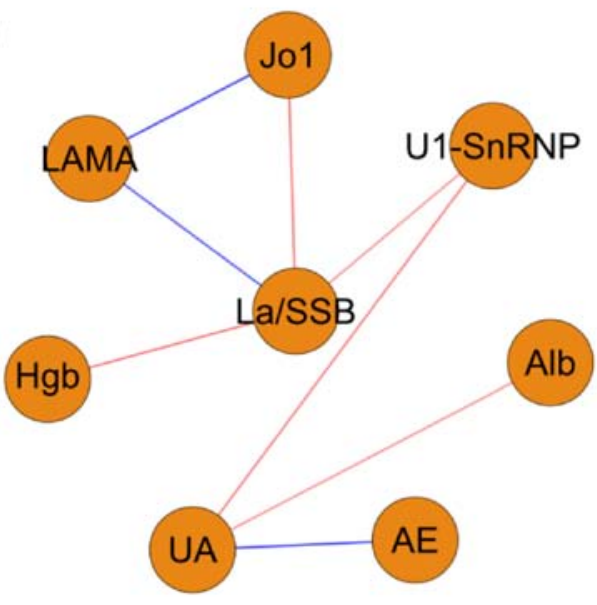

\section{Edge weight $(r):-1=+1$}

Figure 3. Exacerbation-associated modules. (A) Cluster 1; (B) cluster 2; (C) cluster 3; and (D) cluster 4. The size of each node is proportional to its weighted degree value. Correlation coefficients with $\mathrm{P}>0.05$ were filtered out. The colour of each edge indicates the correlation coefficient (edge weight) between two nodes. AE, number of exacerbations in the previous year; Alb, serum albumin; CAT, chronic obstructive pulmonary disease assessment test score; CS, current smoker; Hgb, haemoglobin; Glb, serum globulin; Neu, peripheral blood neutrophil count; sp, sputum; UA, serum uric acid; Sm, Smith antigen; P0, ribosomal phosphoprotein P0; Ro/SSA, Ro/Sjögren syndrome type A antigen; La/SSB, La/Sjögren syndrome type B antigen; Scl70, DNA Topoisomerase I; LAMA, long-acting muscarinic antagonist; Jo1, histidyl-tRNA synthetase; U1-SnRNP, U1 small nuclear ribonucleoprotein; TPO, thyroid peroxidase; PR3, proteinase-3; MPO, myeloperoxidase.

airway/circulating autoantibody responses in heterogenous subgroups of COPD.

A number of previous studies have explored the associations among clinical, functional and biological parameters, and exacerbation risk, in COPD. For example, it was reported that the CAT score can assist in the prediction of COPD exacerbations (38); in the present study, it was observed that the CAT score was significantly associated with retrospective exacerbations only in two subgroups (cluster 1 and cluster 3), suggesting clinical heterogeneity in patients with COPD. Additionally, it was demonstrated that deteriorating airflow limitation is associated with an increasing prevalence of exacerbations (39); however, FEV1 lacks sufficient precision (wide variation) to be used clinically as a predictor of exacerbation in patients with COPD (40). The present study reported that airflow limitations were associated with retrospective exacerbations only in one COPD subgroup, which suggested the heterogeneity of exacerbation risks and was consistent with previous reports. Peripheral neutrophil count represents low-grade systemic inflammation in a number of chronic conditions (41); Hong et al (42) reported that the blood neutrophil count was significantly correlated with main clinical outcomes in patients with COPD. Of note, it was observed in the present study that blood neutrophil count was positively associated with retrospective exacerbation only in one subgroup of COPD patients (cluster 2) and was negatively associated with retrospective exacerbation in another subgroup (cluster 4). These results indicated that the existence of systemic inflammation is also heterogeneous; however, verification of this requires further research. Finally, a previous study reported that serum uric acid was associated with an increased risk of COPD exacerbation (43); 
Table IV. Topological properties of the exacerbation-related module of the four clusters.

\begin{tabular}{lllll}
\hline Factor & Module 1 (Cluster 1) & Module 2 (Cluster 2) & Module 3 (Cluster 3) & Module 4 (Cluster 4) \\
\hline Number of nodes & 4 & 13 & 11 & 8 \\
Average degree & 1.5 & 8.308 & 6.364 & 2 \\
Number of edges & 3 & 54 & 35 & 8 \\
Network diameter & 2 & 2 & 3 & 4 \\
Graph density & 0.5 & 0.692 & 0.636 & 0.286 \\
AE-node degree & 1 & 8 & 5 & 1 \\
AE-correlated nodes & Positive: CAT & Positive: Neu & Positive: CAT & Positive: none \\
& Negative: none & Negative: & sputum anti-PR3 & Negative: UA \\
& sputum anti-P0 & sputum anti-MPO & sputum anti-Ro/SSA \\
& sputum anti-Scl70 & sputum anti-U1-SnRNP
\end{tabular}

AE, number of exacerbations in the previous year; Neu, peripheral blood neutrophil count; CAT, chronic obstructive pulmonary disease assessment test score; Glb, serum globulin; UA, serum uric acid; Sm, Smith antigen; P0, ribosomal phosphoprotein P0; Ro/SSA, Ro/Sjögren syndrome type A antigen; Scl70, DNA topoisomerase I; U1-SnRNP, U1 small nuclear ribonucleoprotein; PR3, proteinase-3; MPO, myeloperoxidase.

however, the present study reported that serum uric acid was negatively associated with retrospective exacerbation in a subgroup of patients, which may be connected to population heterogeneity and/or recall bias during the collection of retrospective information.

Differential module analysis also provided further insight into COPD by demonstrating relationships between autoantibody modules and clinical variables in the various subgroups. Module 1 demonstrated a simple structure where retrospective exacerbations were only associated with CAT score, suggesting that it may be easier to prevent exacerbation in this subgroup (cluster 1). Modules 2 and 3 exhibited complex structures, high network density and high degrees of their respective AE-nodes, pointing towards the complexity and difficulty of preventing exacerbation in these subgroups. Of note, sputum autoantibodies (U1-SnRNP, PR3, and Ro/SSA) were negatively associated with exacerbation risk in module 2 , but were positively related to exacerbation risk in module 3, implying the dual character and heterogeneity of airway autoantibody responses. Thus, these autoantibodies may mediate tissue injury, but may also serve a protective role by removing senescent cells and maintaining immune homeostasis.

The present study had two main strengths. First, autoantibody profiles were detected in sputum and serum simultaneously, whereas the majority of previous clinical studies have detected autoantibodies only in serum or plasma (34,44-47). In this study and a previous preliminary study (13), it was observed that sputum autoantibodies were more clinically relevant than serum autoantibodies, suggesting that studies solely focused on circulating autoantibodies may provide limited information. Second, due to the heterogeneity and complexity of autoantibody responses in cases of COPD, an integrative method was applied based on unsupervised classification. This method differs from previously published COPD autoantibody studies in that it provides the capacity to visualise a wide range of autoantibodies in heterogeneous subgroups, rather than focusing on a single or small number of autoantibodies and viewing all patients as homogenous. Without dividing patients into heterogeneous subgroups, those prior studies may have generated inconsistent findings $(46,47)$.

A number of limitations of the current study should be discussed. First, this was a cross-sectional study, so causal relationships could not be drawn, meaning that exacerbation-associated factors identified in this study should be validated using longitudinal cohort data; however, previous studies reported that the type of inflammatory responses observed during exacerbation may depend on patient phenotype in stable disease (48-52), suggesting that patient parameters in stable disease and exacerbation are closely associated $(3,53)$. Second, as this preliminary study was performed to investigate the heterogeneities of airway/circulating autoantibody responses in patients with COPD, the autoantibody profile data provide limited clinical information to accurately discriminate the COPD subgroups. Third, this study was preliminary and was limited to the analysis of autoantibodies against ten autoantigens. The inclusion of an increased number of diverse autoantibodies may be more clinically informative. Furthermore, autoreactive B cells, which are the source of autoantibodies, should be studied in the future. Finally, the patients recruited into the present study were predominantly male, which may have been related to their risk factors. According to the China Global Adults Tobacco Survey of 2010, 52.9\% of 
males and $2.4 \%$ of females were current smokers (54). In China, cigarette smoking is the main risk factor for COPD, although in rural Southern China it has been replaced by exposure to biomass fuel (55). The patients in the cohort were admitted to a university teaching hospital in Guangzhou (the largest city in Southern China). Thus, cigarette smoking would have been the main risk factor for COPD but would have resulted in a sexual bias, as more males than females are smokers.

In conclusion, using unsupervised clustering and network analysis, it was demonstrated that: i) Pulmonary autoantibody responses were heterogeneous and associated with exacerbation risk in certain subgroups, and therefore their dual character should be taken into consideration in future research; and ii) airway and circulating autoantibody profiles can identify COPD subgroups with various factors associated with exacerbation risk and distinct network topologies. The present study also provides support for future strategies involving personalised predictive biomarker identification and precision management. Further clinical research should focus on local (airway) autoimmune responses.

\section{Acknowledgements}

Not applicable.

\section{Funding}

This study was supported by the National Key R\&D Program of China (grant nos. 2017YFC1310600, 2017YFC1310601 and 2018YFC1311900); Guangzhou Healthcare Collaborative Innovation Major Project (grant no. 201604020012); National Natural Science Foundation of China and Canadian Institutes of Health Research (grant no. 81361128004); Natural Science Foundation of Guangdong Province (grant no. 2015A030310497); Medical Scientific Research Foundation of Guangdong Province (grant no. C2017050); Guangzhou Respiratory Disease Research and Clinical Center Transformation (grant no. 2014Y2-00540); and 111 Project (grant no. D18010).

\section{Availability of data and materials}

All the data generated and analysed in the present study are available from the corresponding author upon reasonable request.

\section{Authors' contributions}

ZL, FL, FW, TP and RC designed the study and drafted the manuscript. ZL, FW, YY, WJ, LZ, JZ, KD, JXX and RC recruited patients and collected clinical data. FL, JX and TP conducted Luminex detection. ZL, FW and YY analyzed microarray data. ZL, XC, WJ, WG, JXX and JZ conducted quality control on the clinical data. ZL and MJ performed data mining. WG and YY processed biological samples.

\section{Ethics approval and consent to participate}

The present study was approved by The Ethics Committee of The First Affiliated Hospital of Guangzhou Medical
University (no. 2017-22). Informed consent was obtained from all patients. The trial registration number for the study was NCT03240315.

\section{Patient consent for publication}

Written informed consent was obtained from the patients for publication of the data included in the present manuscript.

\section{Competing interests}

The authors declare that they have no competing interests.

\section{References}

1. Vogelmeier CF, Criner GJ, Martinez FJ, Anzueto A, Barnes PJ, Bourbeau J, Celli BR, Chen R, Decramer M, Fabbri LM, et al: Global strategy for the diagnosis, management and prevention of chronic obstructive lung disease 2017 report. GOLD executive summary. Am J Respir Crit Care Med 195: 557-582, 2017.

2. Agusti A, Celli B and Faner R: What does endotyping mean for treatment in chronic obstructive pulmonary disease? Lancet 390: 980-987, 2017.

3. Lopez-Campos JL and Agusti A: Heterogeneity of chronic obstructive pulmonary disease exacerbations: A two-axes classification proposal. Lancet Respir Med 3: 729-734, 2015.

4. Agusti A, MacNee W, Donaldson K and Cosio M: Hypothesis: Does COPD have an autoimmune component? Thorax 58: 832-834, 2003.

5. Brusselle GG, Joos GF and Bracke KR: New insights into the immunology of chronic obstructive pulmonary disease. Lancet 378: 1015-1026, 2011.

6. Polverino F, Seys LJ, Bracke KR and Owen CA: B cells in chronic obstructive pulmonary disease: Moving to center stage. Am J Physiol Lung Cell Mol Physiol 311: L687-L695, 2016.

7. Kheradmand F, Shan M, Xu C and Corry DB: Autoimmunity in chronic obstructive pulmonary disease: Clinical and experimental evidence. Expert Rev Clin Immunol 8: 285-292, 2012.

8. Cosio MG, Saetta M and Agusti A: Immunologic aspects of chronic obstructive pulmonary disease. N Engl J Med 360: 2445-2454, 2009.

9. Cosio MG: Autoimmunity, T-cells and STAT-4 in the pathogenesis of chronic obstructive pulmonary disease. Eur Respir J 24: 3-5, 2004.

10. Caramori G, Ruggeri P, Di Stefano A, Mumby S, Girbino G, Adcock IM and Kirkham P: Autoimmunity and COPD: Clinical Implications. Chest 153: 1424-1431, 2018.

11. Wen L, Krauss-Etschmann S, Petersen F and Yu X: Autoantibodies in chronic obstructive pulmonary disease. Front Immunol 9: 66, 2018.

12. Kim WD, Kim WS, Koh Y, Lee SD, Lim CM, Kim DS and Cho YJ: Abnormal peripheral blood T-lymphocyte subsets in a subgroup of patients with COPD. Chest 122: 437-444, 2002.

13. Liang Z, Long F, Deng K, Jian W, Zhou L, Zheng J, Huang A, Cui D, Jin A, Gao Y, et al: Sputum but not circulating autoantibodies associated with exacerbations risk in patients with chronic obstructive pulmonary disease. Am J Respir Crit Care Med 195: A5248, 2017.

14. Diez D, Agusti A and Wheelock CE: Network analysis in the investigation of chronic respiratory diseases. From basics to application. Am J Respir Crit Care Med 190: 981-988, 2014.

15. Barabasi AL, Gulbahce N and Loscalzo J: Network medicine: A network-based approach to human disease. Nat Rev Genet 12: 56-68, 2011.

16. Noell G, Faner R and Agusti A: From systems biology to P4 medicine: Applications in respiratory medicine. Eur Respir Rev 27: pii: 170110, 2018.

17. Watts G: Alvar Agusti: Bringing systems biology to COPD. Lancet 390: 927, 2017.

18. Divo MJ, Casanova C, Marin JM, Pinto-Plata VM, de-Torres JP, Zulueta JJ, Cabrera C, Zagaceta J, Sanchez-Salcedo P, Berto J, et al: COPD comorbidities network. Eur Respir J 46: 640-650, 2015.

19. Grosdidier S, Ferrer A, Faner R, Piñero J, Roca J, Cosío B, Agustí A, Gea J, Sanz F and Furlong LI: Network medicine analysis of COPD multimorbidities. Respir Res 15: 111, 2014. 
20. Faner R, Gutierrez-Sacristan A, Castro-Acosta A, Grosdidier S, Gan W, Sánchez-Mayor M, Lopez-Campos JL, Pozo-Rodriguez F, Sanz F, Mannino D, et al: Molecular and clinical diseasome of comorbidities in exacerbated COPD patients. Eur Respir J 46: 1001-1010, 2015.

21. Noell G, Cosio BG,Faner R, Monsó E, Peces-Barba G, de Diego A, Esteban C, Gea J, Rodriguez-Roisin R, Garcia-Nuñez M, et al: Multi-level differential network analysis of COPD exacerbations. Eur Respir J 50: pii: 1700075, 2017.

22. Global Strategy for the Diagnosis, Management and Prevention of COPD - 2015. Global Initiative for Chronic Obstructive Lung Disease (GOLD), Fontana, WI, 2015. http://goldcopd.org.

23. Miller MR, Hankinson J, Brusasco V, Burgos F, Casaburi R, Coates A, Crapo R, Enright P, van der Grinten CP Gustafsson P, et al: Standardisation of spirometry. Eur Respir J 26: 319-338, 2005.

24. Paggiaro PL, Chanez P, Holz O, Ind PW, Djukanović R, Maestrelli $\mathrm{P}$ and Sterk PJ: Sputum induction. Eur Respir J Suppl 37: 3S-8S, 2002.

25. Bafadhel M, McCormick M, Saha S, McKenna S, Shelley M, Hargadon B, Mistry V, Reid C, Parker D, Dodson P, et al: Profiling of sputum inflammatory mediators in asthma and chronic obstructive pulmonary disease. Respiration 83: 36-44, 2012.

26. Mackay IR, Leskovsek NV and Rose NR: Cell damage and autoimmunity: A critical appraisal. J Autoimmun 30: 5-11, 2008

27. Packard TA, Li QZ, Cosgrove GP, Bowler RP and Cambier JC: COPD is associated with production of autoantibodies to a broad spectrum of self-antigens, correlative with disease phenotype. Immunol Res 55: 48-57, 2013.

28. Hoenderdos $\mathrm{K}$ and Condliffe $\mathrm{A}$ : The neutrophil in chronic obstructive pulmonary disease. Am J Respir Cell Mol Biol 48 $531-539,2013$.

29. Sangaletti S, Tripodo C, Chiodoni C, Guarnotta C, Cappetti B Casalini P, Piconese S, Parenza M, Guiducci C, Vitali C and Colombo MP: Neutrophil extracellular traps mediate transfer of cytoplasmic neutrophil antigens to myeloid dendritic cells toward ANCA induction and associated autoimmunity. Blood 120: 3007-3018, 2012

30. de Hoon MJ, Imoto S, Nolan J and Miyano S: Open source clustering software. Bioinformatics 20: 1453-1454, 2004.

31. Page RD: TreeView: An application to display phylogenetic trees on personal computers. Comput Appl Biosci 12: 357-358, 1996.

32. Bastian M, Heymann S and Jacomy M: Gephi: An open source software for exploring and manipulating networks. International AAAI Conference on Weblogs and Social Media, San Jose, CA p2, 2009.

33. Cheng G, Zhang N, Wang Y, Rui J, Yin X and Cui T: Antibodies of IgG, IgA and IgM against human bronchial epithelial cell in patients with chronic obstructive pulmonary disease. Clin Lab 62: 1101-1108, 2016.

34. Sigari N, Moghimi N, Shahraki FS, Mohammadi S and Roshani D: Anti-cyclic citrullinated peptide (CCP) antibody in patients with wood-smoke-induced chronic obstructive pulmonary disease (COPD) without rheumatoid arthritis. Rheumatol Int 35: 85-91, 2015.

35. Xiong Y, Gao S, Luo G, Cheng G, Huang W, Jiang R, Wang Y and Cui T: Increased circulating autoantibodies levels of IgG, IgA, IgM against cytokeratin 18 and cytokeratin 19 in chronic obstructive pulmonary disease. Arch Med Res 48: 79-87, 2017.

36. Luo XM, Liu XY, Tang JH, Yang W, Ni ZH, Chen QG and Wang X: Autoantibodies against CD80 in patients with COPD. Clin Transl Immunology 5: e103, 2016.

37. Shindi R, Almehairi A, Negm OH, Kalsheker N, Gale NS, Shale DJ, Harrison TW, Bolton CE, John M, Todd I, et al: Autoantibodies of IgM and IgG classes show differences in recognition of multiple autoantigens in chronic obstructive pulmonary disease. Clin Immunol 183: 344-353, 2017.
38. Lee SD, Huang MS, Kang J, Lin CH, Park MJ, Oh YM, Kwon N, Jones PW and Sajkov D; Investigators of the Predictive Ability of CAT in Acute Exacerbations of COPD (PACE) Study: The COPD assessment test (CAT) assists prediction of COPD exacerbations in high-risk patients. Respir Med 108: 600-608, 2014.

39. Mullerova H, Maselli DJ, Locantore N, Vestbo J, Hurst JR, Wedzicha JA, Bakke P, Agusti A and Anzueto A: Hospitalized exacerbations of COPD: Risk factors and outcomes in the ECLIPSE cohort. Chest 147: 999-1007, 2015.

40. Soriano JB, Lamprecht B, Ramirez AS, Martinez-Camblor P, Kaiser B, Alfageme I, Almagro P, Casanova C, Esteban C, Soler-Cataluña JJ, et al: Mortality prediction in chronic obstructive pulmonary disease comparing the GOLD 2007 and 2011 staging systems: A pooled analysis of individual patient data. Lancet Respir Med 3: 443-450, 2015.

41. Liang Z, Liu L, Zhao H, Xia Y, Zhang W, Ye Y, Jiang M and Cai S: A systemic inflammatory endotype of asthma with more severe disease identified by unbiased clustering of the serum cytokine profile. Medicine (Baltimore) 95: e3774, 2016.

42. Hong Y, Park J, Jung YJ, Jeong JS, Kim JH and Kim WJ: Clinical significance of blood neutrophil differential count in patients with COPD. Eur Respir J 50: PA3990, 2017.

43. Bartziokas K, Papaioannou AI, Loukides S, Papadopoulos A, Haniotou A, Papiris S and Kostikas K: Serum uric acid as a predictor of mortality and future exacerbations of COPD. Eur Respir J 43: 43-53, 2014.

44. Wood AM, de Pablo P, Buckley CD, Ahmad A and Stockley RA: Smoke exposure as a determinant of autoantibody titre in $\alpha 1$-antitrypsin deficiency and COPD. Eur Respir J 37: 32-38, 2011.

45. Brandsma CA, Kerstjens HA, Geerlings M, Kerkhof M, Hylkema MN, Postma DS and Timens W: The search for autoantibodies against elastin, collagen and decorin in COPD. Eur Respir J 37: 1289-1292, 2011.

46. Greene CM, Low TB, O'Neill SJ and McElvaney NG: Anti-proline-glycine-proline or antielastin autoantibodies are not evident in chronic inflammatory lung disease. Am J Respir Crit Care Med 181: 31-35, 2010.

47. Lee SH, Goswami S, Grudo A, Song LZ, Bandi V, Goodnight-White S, Green L, Hacken-Bitar J, Huh J, Bakaeen F, et al: Antielastin autoimmunity in tobacco smoking-induced emphysema. Nat Med 13: 567-569, 2007.

48. Hurst JR: Exacerbation phenotyping in chronic obstructive pulmonary disease. Am J Respir Crit Care Med 184: 625-626, 2011.

49. Bhowmik A, Seemungal TA, Sapsford RJ and Wedzicha JA Relation of sputum inflammatory markers to symptoms and lung function changes in COPD exacerbations. Thorax 55: 114-120, 2000.

50. Saetta M, Di Stefano A, Maestrelli P, Turato G, Ruggieri MP, Roggeri A, Calcagni P, Mapp CE, Ciaccia A and Fabbri LM: Airway eosinophilia in chronic bronchitis during exacerbations. Am J Respir Crit Care Med 150: 1646-1652, 1994.

51. Bafadhel M, McKenna S, Terry S, Mistry V, Reid C, Haldar P, McCormick M, Haldar K, Kebadze T, Duvoix A, et al: Acute exacerbations of chronic obstructive pulmonary disease: Identification of biologic clusters and their biomarkers. Am J Respir Crit Care Med 184: 662-671, 2011.

52. Gao P, Zhang J, He X, Hao Y, Wang K and Gibson PG: Sputum inflammatory cell-based classification of patients with acute exacerbation of chronic obstructive pulmonary disease. PLoS One 8: e57678, 2013

53. Miravitlles M, D'Urzo A, Singh D and Koblizek V: Pharmacological strategies to reduce exacerbation risk in COPD: A narrative review. Respir Res 17: 112, 2016.

54. GH Y: Global adult tobacco survey (GATS) China 2010 country report. China Sanxia Press, Beijing, China, 2011.

55. Liu S, Zhou Y, Wang X, Wang D, Lu J, Zheng J, Zhong N and Ran P: Biomass fuels are the probable risk factor for chronic obstructive pulmonary disease in rural South China. Thorax 62: 889-897, 2007. 